\title{
Authentication by Touch Operation on Smartphone with Support Vector Machine
}

\author{
Nozomi Miyamoto ${ }^{1}$, Chihiro Shibata ${ }^{2}$, Toshiyuki Kinoshita ${ }^{2}$ \\ ${ }^{1}$ Lac Co. Ltd., Chiyoda Tokyo, Japan \\ ${ }^{2}$ Tokyo University of Technology, Hachioji Tokyo, Japan
}

\begin{abstract}
In this research, we proposed a method to apply Support Vector Machine (SVM) for personal authentication by touch operation on smartphone. The SVM is one of machine learning that generates a maximum margin separation line furthest from both of the separated sample point clusters. The data is divided into training data and test da$t a$, and the authentication accuracy is measured by applying Cross Validation. For four kinds of touch operations: single tap, double tap, swipe, rotation, and six pairs of these touch operations, we compared the authentication rates of each touch operation and pair of operations and measured how the authentication rate changes along with the number of training data and the number of test data.

The experimental results show that the authentication rate is as low as 60 to $85 \%$ for a single touch operation, but when the pair of touch operations are applied, the authentication rate reaches 95 to $100 \%$ at the maximum. It can be used sufficiently for personal authentication. As the number of registered data and test data increases, the authentication rate also increases. However, considering user's burden at registration and the registration time, it is considered that the combination of the double tap and swipe, 20 registered data and 2 test data are optimal in practical use.
\end{abstract}

\section{Introduction}

Nowadays, smartphones are becoming more common in daily life just like computers, telephones, databases, cameras, locators and many others. The personal information such as password, telephone directory, health information and corporate information is usually stored in smartphone. In order to protect this information, identity authentication is performed prior to the login process. Password authentication such as number password, pattern base password, PIN base password, and biometric recognition is used for personal authentication.

PIN-based and pattern-based passwords are frequently used on most smartphones as they are easy to remember and convenience. However, existing research showed that even though PINbased passwords are easy to remember, they also become an easy target as it is weak against attack, meanwhile, pattern passwords can be easily broken using shoulder hacking attacks. Attackers usually use accelerometers and gyroscopes as methods to predict passwords.

The problems of these conventional authentication methods are being solved by using biometric authentication. In this research, we aim to investigate the accuracy of personal identification using individual behavioral features in touch operation of the smartphone. Behavioral features of touch operations are unique to individuals and they cannot be stolen, lost or forgotten

Besides, by using touch operation authentication, it is possible to prevent spoofing. If the touch operation of the user of the smartphone does not match with the registered principal, the user is blocked from getting the access. With this, it is possible to prevent person impersonation.

Authentication by the touch operation is similar to the typing authentication which uses behavioral features of the user hitting the keyboard. Typing authentication analyzes behavioral features such as custom habitual typing rhythm and strength of users. On the other hand, the touch operation analyzes the behavioral features when the user touches the screen of the smartphone (see Figure 1). 


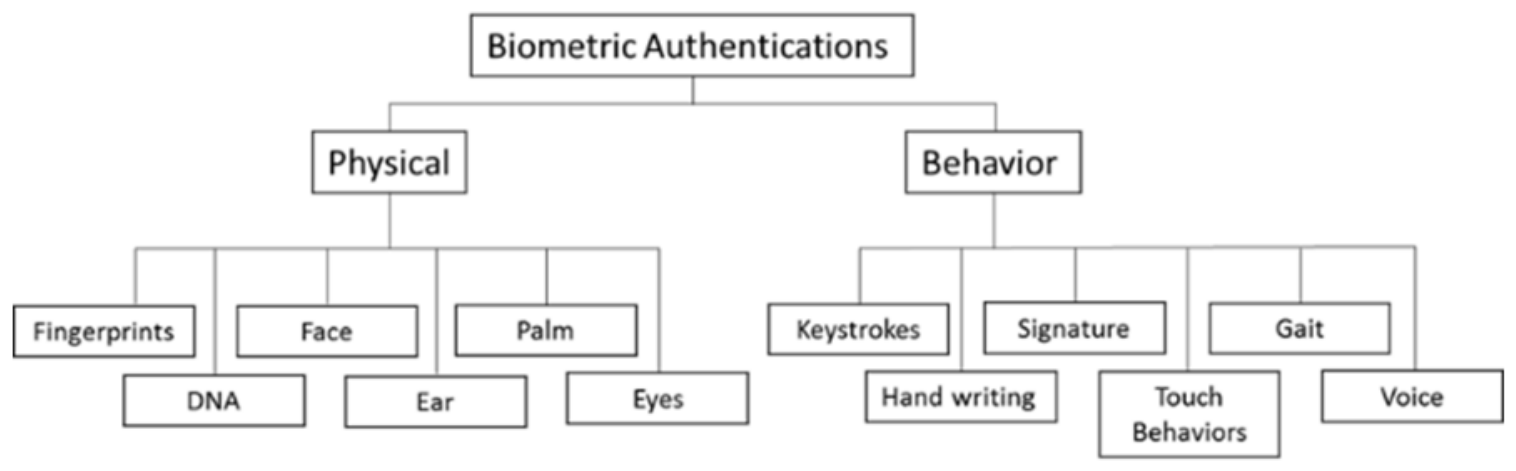

Figure 1. Types of biometric authentication

In this research, we propose authentication using Support Vector Machine (SVM) which is one of the machine learning methods to improve the authentication accuracy. The objectives of this research are summarized as follows.

(1) Verify the authentication accuracy when the user authenticates using the following touch operation.
(a) Single tap
(b) Double tap
(c) Swipe
(d) Rotation

(2) Improve authentication accuracy by using Support Vector Machine (SVM) which is one of machine learning.

\section{Touch operation authentication}

\subsection{Biometrics}

Biometric authentication provides more secure authentication than traditional password-based methods. It is naturally easy for users to use, and authentication can be performed at high speed. Biometric authentication basically uses human physical or behavioral features [8]. For biometric authentication, various parts or behavioral characteristics of a person can be used. Figure 1 shows the types of biometric authentication. The application conditions of human behavioral characteristics for biometric authentication are shown in Table 1 [9].

There are two kinds of biometrics, verification system and identification system. The verification system verifies whether the user who is currently operating the device is the principal or not by comparing the touch operation data with the data of the principal which has been registered in the smartphone.
Table 1. Biometric Authentication Application Conditions

\begin{tabular}{|c|l|}
\hline Uniqueness & $\begin{array}{l}\text { People have their own unique- } \\
\text { ness that can be used to distin- } \\
\text { guish each action }\end{array}$ \\
\hline Universality & $\begin{array}{l}\text { People seem to have the same } \\
\text { behaviour, which in reality are } \\
\text { difference }\end{array}$ \\
\hline Persistence & $\begin{array}{l}\text { Age affects human behaviour } \\
\text { such as the elderly are slow to } \\
\text { react compared to the young } \\
\text { people }\end{array}$ \\
\hline Availability & $\begin{array}{l}\text { Modern technology can repro- } \\
\text { duce the human behaviour. }\end{array}$ \\
\hline Performance & $\begin{array}{l}\text { Biometric authentication built } \\
\text { using touch operation show ac- } \\
\text { curacy, speed, and robustness }\end{array}$ \\
\hline Acceptability & $\begin{array}{l}\text { Acceptance of technology in } \\
\text { common daily life }\end{array}$ \\
\hline Reproducibility & $\begin{array}{l}\text { The ability to configure authen- } \\
\text { tication system }\end{array}$ \\
\hline
\end{tabular}

For example, when a fingerprint is pressed on the authentication machine, the verification system determines whether the fingerprint matches the information of the principal registered in the database.

The identification system attempts to identify an unknown person or an unknown organism. Since the identification system tries to identify an unknown user, it is necessary to collate all information of current user's data and store it in the database. The identification system is described as $1: n$ collation ( $n$ is the total number of biometric information registered in the database). 


\subsection{Touch operation authentication on smartphone}

Touch operation is a pointing operation performed by touching the display with a finger, a pen, etc. Touch operation can be operated by directly touching icons or buttons on the screen with fingers. Rather than operating using a pointing device such as a mouse, more intuitive operation is possible. The user interface designed under the premise of the touch operation is called "touch interface". When operating with smartphones, "multi-touch interface" which implement a complex operation such as enlargement/reduction of a screen by touching it with multiple fingers become more common.

In this research, we verified the accuracy of authentication by touch operation. Authentication by touch operation refers to a method of discriminating whether a person is the principal or not based on the behavioral characteristics of a user's habit in touch operation. By focusing on the individual difference of the touch operation of the finger, we can identify the principal by determining whether the user fitted to the condition of the motion characteristic. The characteristic of the touch operation is the similarity between registered data and the input data at the time of verification is calculated with regards to the contact time and coordinates of the contact point between the finger and the touch panel.

\section{Touch operation}

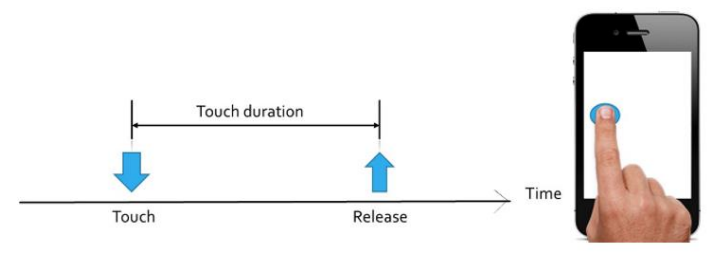

Figure 2. Single tap

This research focuses on the touch operation of browsing which is one of the basic functions of many smartphone applications and targeted the following touch operations.

\subsection{Single tap operation}

Single tap is frequently used to activate screen elements like button operation, and is also used to cancel or escape current tasks such as dialogs and menu. In single tap operation, duration of touch is

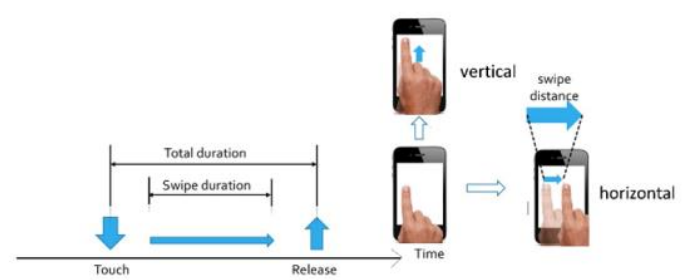

Figure 3. Double tap

measured. This is the time from when the finger touches the screen until it separates.

\subsection{Double tap operation}

The double tab is operated by quickly tap the screen twice without moving the finger like double-clicking while using the computer. Double tap is usually used when selecting items and text, or when expending contents. In double tap operation, three parameters; the duration of the first tap, the interval time between the first and second taps, and the second tap duration, are measured.

\subsection{Swipe operation}

Swipe is operated by moving the finger up or down or left or right while touching the entire screen or a wide range of screen. Swipe operation is used to move the screen or turn the page. In swipe operation, the time and distance traveled while the finger is moving and the duration of the contact is among the three parameters measured; the time when the finger is in contact with the screen, the time when the finger is moving, and the movement distance.

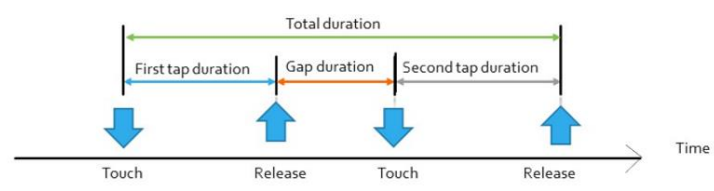

Figure 4. Swipe

\subsection{Rotation operation}

Rotation is used to rotate items. The user rotates clockwise or counter-clockwise while touching the finger on the screen. In the rotation, the duration of rotation and the angle of rotation are measured.

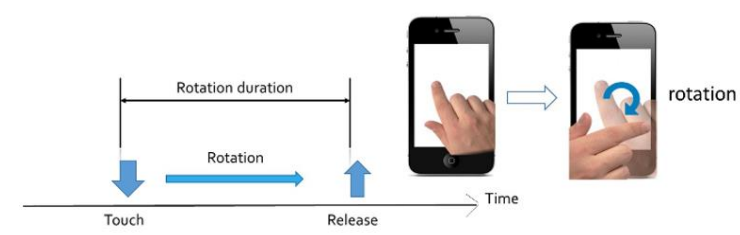

Figure 5. Rotation 


\section{Authentication method}

\subsection{Support Vector Machine（SVM）}

SVM is a method of generating maximum margin separation lines that are the furthest from both of the separated sample point clusters when the set of sample points can be separated by a straight line in the space. At that time, a maximum margin separation line is obtained for several sample points in advance (teacher data). When a new sample point is added, a maximum margin separation line including the new sample point is generated by learning from the teacher data. This way, it is possible to determine which cluster the newly added sample point belongs to. Therefore, SVM is one of the supervised learning of machine learning.

When there are two-dimensional data with two type of characteristics, a straight line of $y=a x+b$ is drawn from positive and negative given learning data. Based on the straight line, we can identify whether the test data is positive or negative (red is positive and blue is negative). SVM finds the most distant region (maximum margin) between the two groups and draws an identification line at the center (see Figure 6).

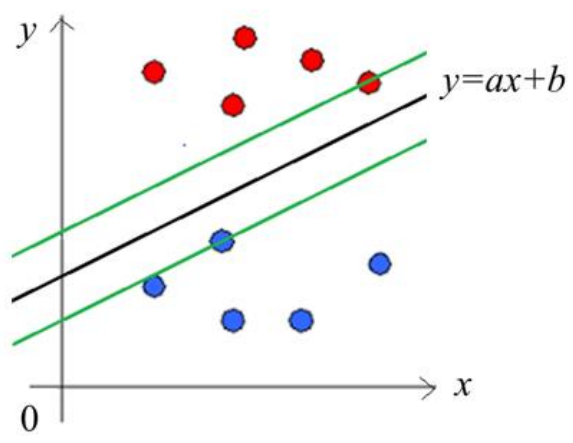

Figure 6. Support Vector Machine

\subsection{Cross validation}

Data is divided into training data and test data and authentication accuracy are measured. For example, in case of preparing 100 pieces of data, the data is divided into 5 groups with 20 pieces each. During the first verification, one group is evaluated as test data and the other as learning data to measure authentication accuracy. In the second verification, a group different from the first one is used as test data, and the third verification used a group different from the first and the second times as the test data. This is done to all five groups and an average of the accuracy is taken (see Figure 7). As a result, it is confirmed that the learning effect increases when data increases.

\subsection{FRR, FAR}

In biometric authentication, it is indicated as a False rejection rate (FRR) and a False acceptance rate (FAR) as evaluation indicators. FRR is the rate of refusal that the principal is rejected by mistake despite attempting to authenticate himself, and is calculated as follows.

$$
F R R=\frac{R}{N}
$$

$R$ : Number of times the principal is rejected by mistake

$N$ : Number of times the principal tried to authentication

The smaller the FRR value is, the less likely a legitimate user is rejected by mistake, indicating that the authentication accuracy is high.

On the other hand, the FAR is the rate at which another person is accidentally recognized as the principal when attempting to authenticate, and is calculated as follows.

$$
\begin{aligned}
& F A R=\frac{A}{N} \\
& A: \text { Number of times the non-principal is mis- } \\
& \text { judged as a principal } \\
& N: \begin{array}{l}
\text { Number of times the principal tried to } \\
\text { authentication }
\end{array}
\end{aligned}
$$

Like FRR, the smaller the value of FAR, the less likely the other person is mistakenly accepted as the principal, indicating that the safety of the system is high.

Both FRR and FAR show that the smaller the value, the higher the authentication accuracy. In most cases, however, these two indicators are in a trade-off relationship. Usually, when FRR is high, FAR will be low and vice versa. Because it is more damaging to accept others by mistake than being rejected by himself/herself, it is more likely to increase FRR at the expense of FAR. These relationships are shown in Figure 8.

\subsection{Precision, Recall}

The following judgment result may occur in person authentication.

TP (True Positive): The number of times the principal was correctly judged as the principal. 


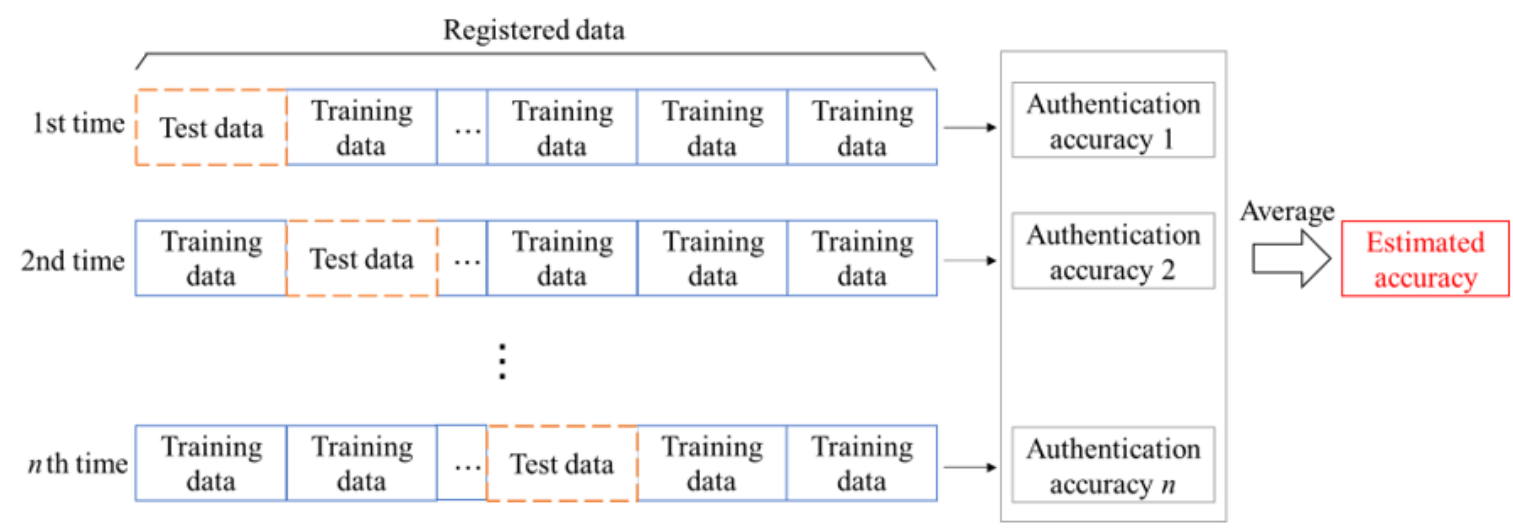

Figure 7. Cross validation

FP (False Positive): Number of times that the nonprincipal is misjudged as the principle.

TN (True Negative): Number of times that the non-principal is correctly judged as the nonprinciple.

FN (False Negative): Number of times that the principal is misjudged as the non-principle.

As the evaluation index, the following values are used.

Precision: The ratio between among those judged to be principals and of those who are actually the principals.

$$
\text { Precision }=\frac{T P}{T P+F P}
$$

Recall: The ratio between those who are actually the principals and judged to be himself / herself.

$$
\text { Recall }=\frac{T P}{T P+F N}
$$

Therefore, Precision corresponds to " 1 -FAR", Recall corresponds to " $1-$ FRR".

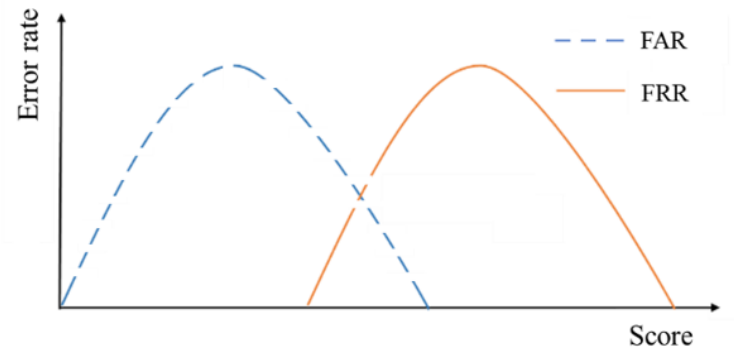

Figure 8. Relationship between FRR and FAR

\section{Related studies}

In previous studies, Yuji Watanabe et al. [14] conduct a research on authentication of combinations touch operations. The main touch operations are flick and drag touch operations. These touch operations were recorded on smartphones using text browsing applications. Additional characteristic manipulation characteristics such as touch area distribution, speed, etc. were also recorded.

Akira Fujita et al. [15] developed an application for measuring contact behavior. In addition to text browsing application, web browsing and image manipulation were also used. In this research, the touch operation is used for reduction and enlargement. From there, the amount of movement of the finger, the moving speed of the finger, and the moving angle was calculated. These studies conclude that it is necessary to increase the number of subjects in order to obtain better results. Furthermore, it also states that the touch operation is not limited to flick, drag, pinch operation. Therefore, in this study, we planned to examine single tap, double tap, swipe, rotation operation, and conduct experiments on 10 test subjects.

\section{Experiment program}

Figure 9 shows the procedure of the proposed authentication system. First, the user (test subject) registers to the system. Registered users save behavioral features in the database on four types of touch operations: single tap, double tap, swipe, and rotation. Next, one or two types of touch operations out of these four types are input as test operations and collated with registered data. Collation is performed by the algorithm of SVM by machine learning. 


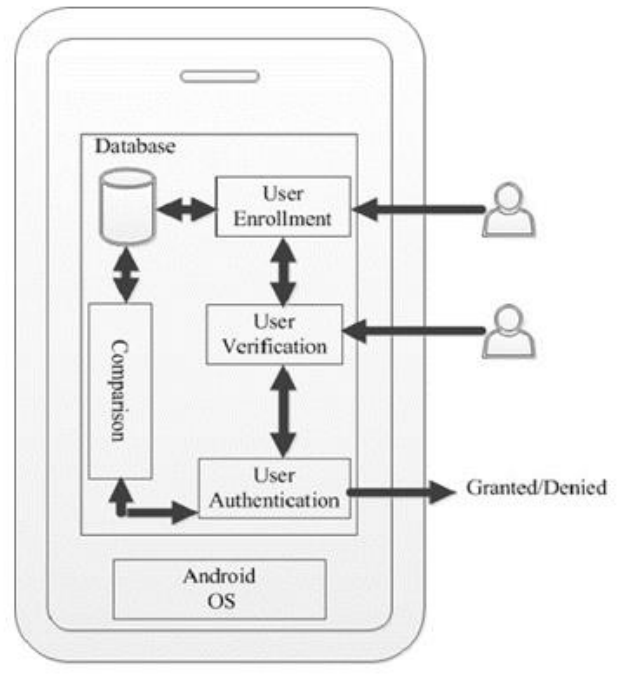

Figure 9. Procedure of proposed authentication

Figure 10 (a) shows the user interface of the registration and authentication system. To register a user, key in the "NEW PROFILE" button on the touch screen and the database of the user as shown in Figure 10 (b). Figure 11 shows authentication of behavioral features in the touch operation of single tap and swipe operation, which information is held in the database of the system. of single tap and swipe operation, which information is held in the database of the system.

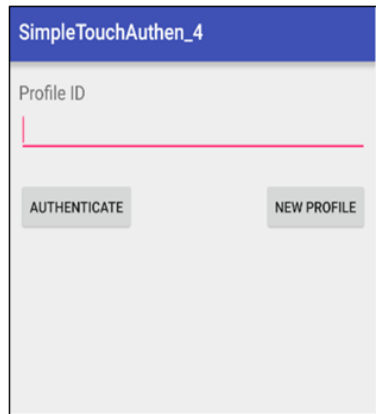

(a) User Interface

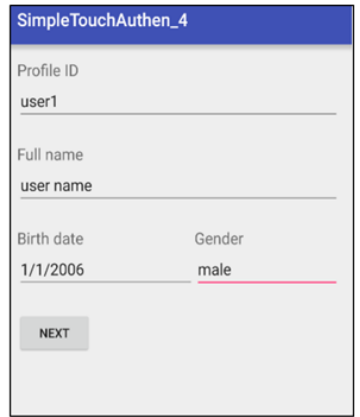

(b) User Database
Figure 10. User Interface and User Database

The authentication system, as shown in Figure 11 , the user first enters the profile ID and touches the "AUTHENTICATE" button of the user interface. Next, behavioral features by touch operation for verification are registered, and the authentication system performs verification.
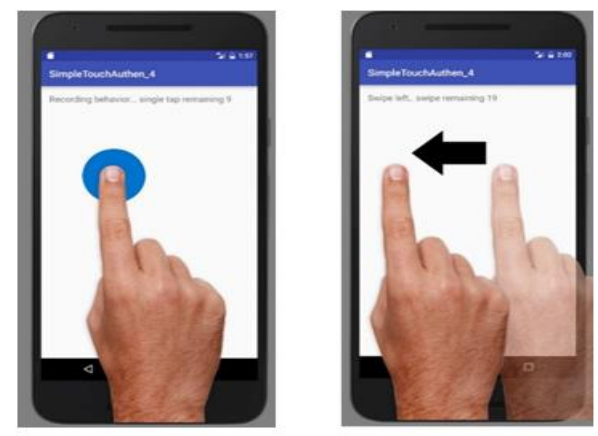

Figure 11. Single tap and swipe verification system

The result of the operation on the touch screen is compared with a user database that plays an important role to determine that the reference threshold biometrics is a registered user.

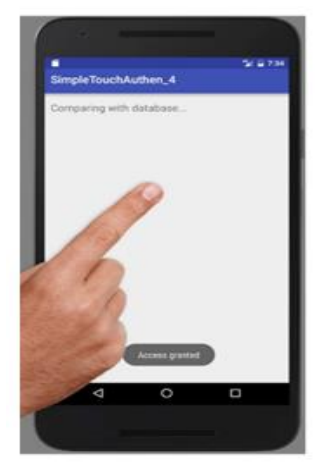

Figure 12. Authentication system

Figure 12 shows an indication of access granted or access denied when the user completes the authentication protocol.

\section{Experiment}

\subsection{Experimental procedures}

In this study, we recruited 10 subjects for research experiments. Subjects were asked to perform the following touch operation.

1. Have each user operate the touch operation twenty times as registration data.

2. Apply SVM to the registration data, and learn characteristics of the touch operation of the principal.

3. Operate each touch operation once or twice as test data.

4. Apply SVM learned on data registered in test data to identify the principal. 


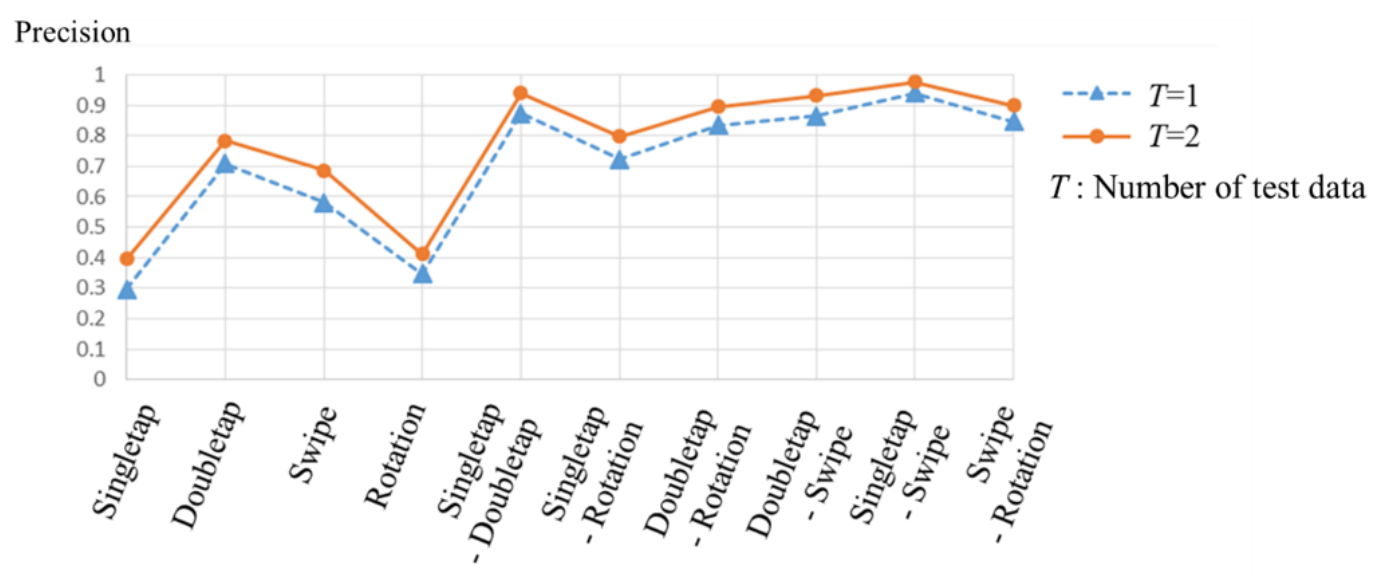

Figure 13. Comparison of touch operations (Precision)

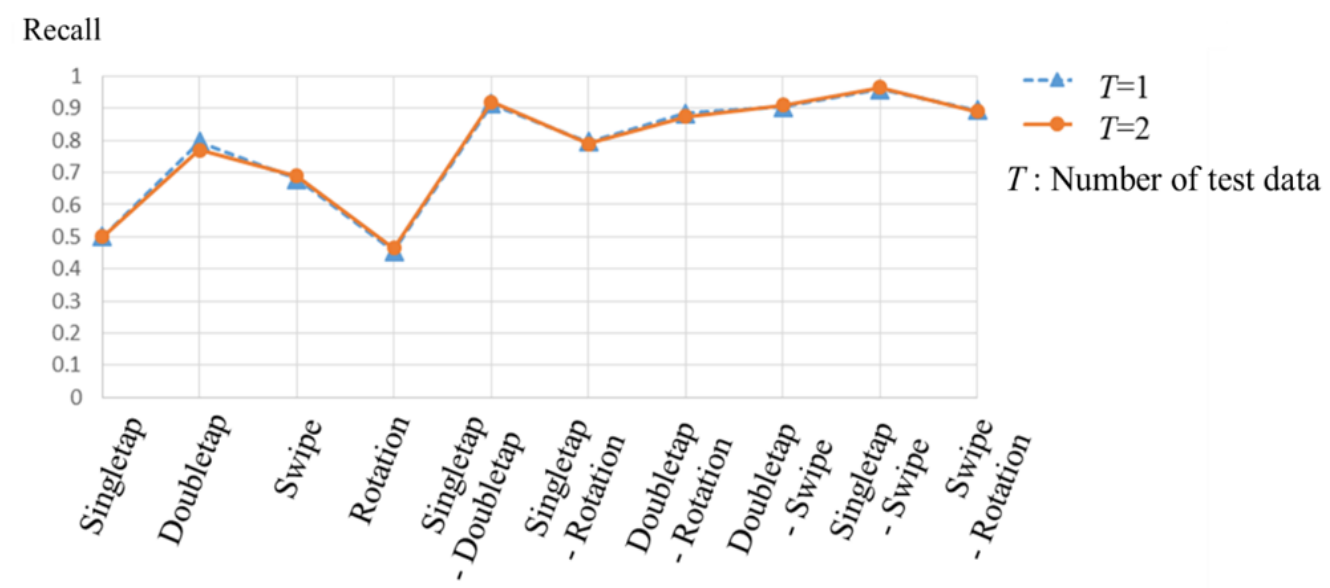

Figure 14. Comparison of touch operations (Recall)

\subsection{Touch operations comparison}

7.2.1. Measurement method. Ten subjects operate four operations of single tap, double tap, swipe, rotation 20 times each. They also performed combine operation of six operations (Single tap - Double tap, Single tap - Swipe, Single tap - Rotation, Double tap - Swipe, Double tap - Rotation, Swipe - Rotation).

In this way 200 data can be obtained for each person $(=(1$ type from the four operations +2 types from the six combined operations) $\times 20$ times), resulting in a total of 2,000 registered data. Cross-validation is used to extract two pieces of data from the registration data and taken as test data. By using SVM for this test data, it identifies whether it is the principal or another person and obtains an authentication rate.

\subsubsection{Results}

For Precision, double tap was the highest in single operations with $78.4 \%$. However, when two kinds of operations were combined, almost all accuracy showed $90 \%$ or more with the combination of double tap - rotation has the highest authentication rate of $97.7 \%$. This indicates that the authentication accuracy is greatly improved (see Figure 13)

The authentication rate is slightly higher for combination of two operations when compared to single operation with $0.7 \%$ difference.

Similar to Precision, Recall also showed higher authentication rate when combining two kinds of operations, while for the number of test data, the authentication rate for Recall did not show 


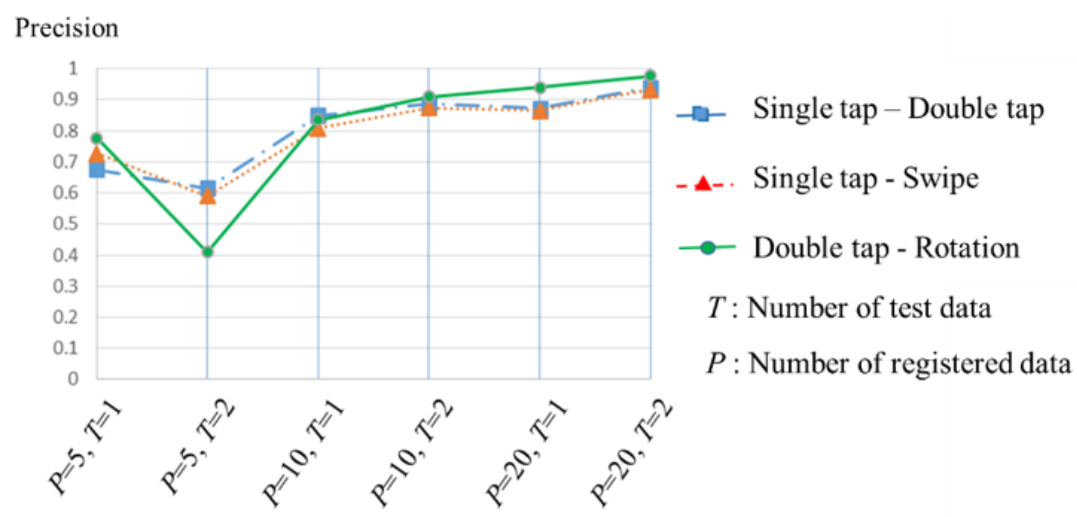

Figure 15. Comparison of the number of registered data and test data (Precision)

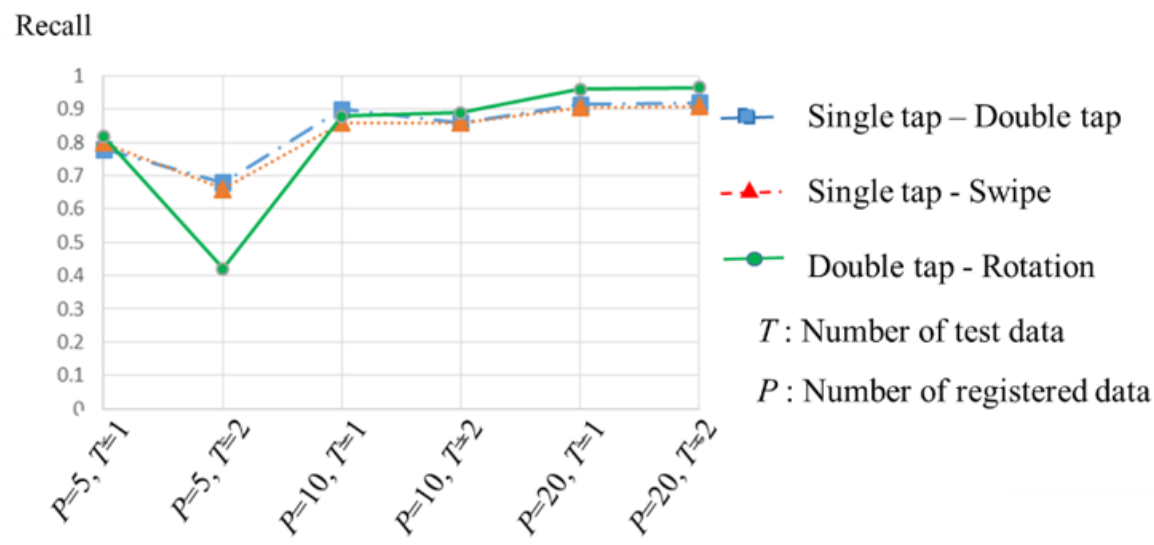

Figure 16. Comparison of the number of registered data and test data (Recall)

significant differences for single operation or combined operations (see Figure 14).

\subsection{Comparison of the number of registered data and the number of test data}

\subsubsection{Measurement conditions}

Based on previous section, the authentication rate between the number of registered data and the number of test data in single tap - double tap, double tap - swipe, double tap - rotation which authentication rate is $93 \%$ or more are compared in Precision and Recall.

\subsubsection{Results}

As the number of registered data increased to 5 , 10 and 20, the authentication rate for both precision and recall increases. When the number of registered data is 20 and the number of test data is 2 , the authentication rate is the highest (see Figures 15 and 16).

\section{Discussion}

Based on the results of the experiment, of the combinations between two kinds of operations, the authentication rate at the time of Single tap - Double tap, Double tap - Swipe, Double tap - Rotation is high. It also shows that the larger the number of registered data, the higher the authentication rate. On the other hand, this experiment confirmed that the authentication rate is higher when two test operations were used instead of one test operations. However, increasing the number of registered data or test data increases the burden on the user and the time required for authentication. For these reasons, the combination of Double Tap - Swipe, 20 registered data, and 2 test operations is considered optimal. 


\section{Conclusion}

This study confirmed the effectiveness of applying SVM to person authentication using behavioral features of fingers by touch operation on smartphone. The combination of Double tap Swipe, 20 registered data, and 2 test operations is considered optimal practically. In the future, it is necessary to improve the performance so that the authentication using touch operation can be handled (Precision). Besides, we also would like to examine the effects of other data analysis methods using artificial intelligence other than SVM.

\section{Reference}

[1] D. Goldman, "Your smartphone will run your life. CNN Money, http://money.cnn.com/2010/10aa/19/technology/smartphones/10 Dec. 2016.

[2] A. J. Aviv, K. Gibson, E. Mossop, M. Blaze and J. M. Smith, "Smudge attacks on smart phone touch screens," Proc. 4th USENIX Conf. Offensive Technol Washington DC, USA, pp.1-7, 2010.

[3] N. H. Zakaria, D. Griffiths, S. Brostoff and J. Yan, "Shoulder surfing defense for recall-based passwords," Proc. 7th Symp. Usable Privacy Secure, Pittsburg, PA, USA, pp.1-12, 2011.

[4] Z. Xu, K. Bai, and S. Zhu, "Tap Logger: Inferring user inputs on smartphone touch-screens using onboard motion sensors," in Proc. 5th ACM Conf. Security Privacy Wireless Mobile Network, pp.113-124, 2012.

[5] E. Owusu, J. Han, S. Das, A. Perrig, and J. Zhang, "ACCessory: Password inference using accelerometers on smartphones," Proc. 12th Workshop Mobile Computer System Application, pp. 9-14, 2012.u

[6] A. K. Jain, A. Ross, and S. Pankanti, "Biometrics: A Tool for Information Security," IEEE Transactions on Information Forensics and Security, Vol. 1, No. 2, pp.125143, 2006.

[7] A.K. Jain, A. Ross, S. Prabhakar, "An Introduction to biometric recognition," IEEE Trans-actions on Circuits and Systems for Video Technology, 14(1): 4-20, 2004.

[8] Raid R. Al-Nima, "Human Authentication with Earprint for Secure Telephone System," IJCCCE, Vol. 12, No. $2,2012$.

[9] Dasari Nag.a Shailaja, "A Simple Geometric Approach for Ear Recognition,” June 2006.

[10] Chao Shen, Yong Zhang, Xiaohong Guan, and Roy A. Maxion, "Performance Analysis of Touch -Interaction Behavior for Active Smart-phone Authentication," IEEE Transactions on Information Forensics and Security, Vol. 11, No. 3, March 2016.
[11] Sanraj Rajendra Bandre, "Design and Implementation of Smartphone Authentication System based on Colorcode," International Conference on Pervasive Computing (ICPC), 2015.

[12] Nabilah Shabrina, Saiful Akbar and Peb Ruswono Aryan, Palmprint, "Authentication in Smart phone Using Phase-Only Correlation Method," ICACSIS, 2013.

[13] "Gestures", https://material.io/guidelines/pat terns/ gestures.html/gestures-touch-mechanics, 5 August 2016.

[14] Yuji Watanabe, Shunta Ichikawa, "Consideration of Continuous Personal Identification System Using Characteristics of Touch Operation on Smartphones," Computer Science Symposium 2012, pp.797-804.

[15] Akira Fujita, Yuji Watanabe, "Development of applications for personal authentication using features of touch operation on Android terminals," Computer Security Symposium 2013, pp.688-694.

\section{Acknowledgements}

This paper is a summary of the research results that were written at the School of Computer Science, Tokyo University of Technology. Firstly, I would like to express my sincere gratitude to my academic supervisor Dr. Toshiyuki Kinoshita who gave me the opportunity to conduct this research. His guidance from the beginning helped me to complete this paper. Besides my supervisor, my sincere thanks also go to Senior Assistant Professor Chihiro Shibata of the same department for the advice and guidance throughout this paper. Last but not the least, I would like to thank my fellow labomates for the stimulating discussions while carrying out research. 